\title{
Large eddy simulation of pollen transport in the atmospheric boundary layer
}

\author{
Marcelo Chamecki ${ }^{\mathrm{a}}$, Charles Meneveau ${ }^{\mathrm{b}, *}$, Marc B. Parlange ${ }^{\mathrm{c}}$ \\ a Department of Geography and Environmental Engineering, Center for Environmental and Applied Fluid Mechanics, Johns Hopkins University, Baltimore, MD, USA \\ ${ }^{\mathrm{b}}$ Department of Mechanical Engineering, Center for Environmental and Applied Fluid Mechanics, Johns Hopkins University, Baltimore, MD, USA \\ 'School of Architecture, Civil, and Environmental Engineering, Ecole Polytechnique Fédérale de Lausanne, Lausanne, Switzerland
}

\section{A R T I C L E I N F O}

\section{Article history:}

Received 11 April 2008

Received in revised form

25 September 2008

Accepted 10 November 2008

\section{Keywords:}

Pollen

Large eddy simulation

Turbulent dispersion

Ragweed

Heavy particles

\begin{abstract}
A B S T R A C T
This paper presents a framework to simulate pollen dispersal by the wind based on the large eddy simulation (LES) technique. Important phenomena such as the pollen emission by the plants and the ground deposition are parameterized by the lower boundary condition. The numerical model is validated against previously published experiments of point source releases of glass beads and pollen grains in the atmospheric boundary layer. The numerical model is used together with experimental data of pollen emission and downwind deposition from a natural field obtained near Washington, DC, in the summer of 2006. The combined analysis of experimental and numerical data allows to elucidate the emission/transport/deposition process in considerable detail. In particular, the relative fractions of pollen deposited inside the source field and airborne at the edge of the field can be quantified. The use of LES allows quantification of important intermittent deposition events far from the source field.
\end{abstract}

(C) 2008 Elsevier Ltd. All rights reserved.

\section{Introduction}

Dispersion of pollen by the wind is a subject of interest in many fields such as ecology, agronomy and allergology. There are several important questions related to pollen dispersal that have gained attention in the recent years. The development of genetically modified crops has raised questions about cross-pollination and possible contamination of natural fields (e.g. Aylor, 2002; Aylor, Schultes, \& Shields, 2003). In the context of landscape fragmentation, it is often important to determine whether small populations are genetically isolated or if they are able to exchange genetic information with larger (genetically diverse) neighboring populations (Honnay, Jacquemyn, Bossuyt, \& Hermy, 2005). In all these areas, quantitative estimates of the threedimensional plume of airborne pollen grains and consequent ground deposition from an emitting field are required, and a few field experiments have been designed to study this problem (e.g. Jarosz et al., 2003; McCartney \& Lacey, 1991; Raynor, Hayes, \& Ogden, 1972; Raynor, Ogden, \& Hayes, 1970, 1972). However, the results are expected to depend upon several factors, such as plant pollen emission rates, aerodynamic properties of the pollen grains, geometric properties of the field, topography, local vegetation, wind conditions, atmospheric stability, etc. As a consequence, while field experiments are well suited to provide some information on pollen transport mechanisms they are limited to specific field and weather conditions. Numerical simulations do not have this particular drawback and can be a useful supplementary tool to study pollen dispersal in a variety of controlled configurations.

In order to be successful, numerical simulations of pollen dispersal must account for three complex phenomena: pollen emission, transport and deposition. The pollen emission represents the source of pollen in the simulation and in reality is a combination of the pollen release by plants and the pollen entrainment by turbulence. The transport of pollen requires a good representation of the wind field (usually disturbed by the canopy) and must account for the gravitational settling of pollen

\footnotetext{
* Corresponding author. Tel.: +14105167802.

E-mail addresses: chamecki@jhu.edu (M. Chamecki), meneveau@jhu.edu (C. Meneveau), Marc.Parlange@epfl.ch (M.B. Parlange).
} 
grains. The deposition process represents the sink of pollen in the simulation and is a combination of many processes such as gravitational settling, turbulent diffusion and impact with the canopy elements.

Application of numerical simulations to dispersion of pollen has been recently reported by Helbig, Vogel, Vogel, and Fiedler (2004), Jarosz, Loubet, and Huber (2004), Dupont, Brunet, and Jarosz (2006) and Arritt et al. (2007). Helbig et al. (2004) developed a three-dimensional Eulerian model for regional scale prediction using the KAMM/DRAIS mesoscale model. Most of their work was focused on specifying emission, deposition and resuspension fluxes at mesoscale grid resolutions. The main conclusion of their simulations is the large increase in dispersal distances caused by topography and heterogeneous land use.

Jarosz et al. (2004) used a two-dimensional Lagrangian stochastic model to simulate the dispersion of corn pollen from the field experiment performed by Jarosz et al. (2003). The velocity field was parameterized by first- and second-order moments obtained from the Monin-Obukhov similarity theory. The pollen release rate is calibrated to reproduce the concentration profiles measured $3 \mathrm{~m}$ downwind of the edge of the source field using the approach of Aylor and Flesch (2001). Although reasonable agreement was found for the vertical concentration profile $10 \mathrm{~m}$ downwind, the authors reported a significant underprediction of deposition within the first $10 \mathrm{~m}$ and suggested that the reason was the poor representation of turbulence on the transition between the corn field and the bare soil region downwind.

Dupont et al. (2006) coupled a pollen dispersion model to a numerical model of turbulence. The velocity field was calculated following the Reynolds-averaged Navier-Stokes (RANS) approach. Pollen concentrations were represented in an Eulerian framework, through an advection-diffusion equation. Two-dimensional simulations for the field experiments of Jarosz et al. (2003) and Jarosz, Loubet, Durand, Foueillassar, and Huber (2005) were performed. Again a reasonable agreement for the concentration profile was found and the underprediction of the ground deposition was the main problem.

Arritt et al. (2007) used a three-dimensional Lagrangian stochastic model to simulate the dispersion of corn pollen. The velocity field was represented by statistics as in Jarosz et al. (2004). The agreement between measured and predicted deposition was reasonable. The authors listed the possible heterogeneity of the source and computational constraints in the number of particles as the main reasons for the discrepancies.

In this paper we describe tools and frameworks to simulate pollen dispersal using large eddy simulation (LES). The use of LES allows for an accurate representation of the wind field. Pollen concentrations are simulated in an Eulerian framework using an advection-diffusion equation, and the important processes of pollen release and ground deposition are parameterized in a consistent way through the lower boundary condition. The mathematical model and numerical discretization are described in Section 2 and validated against classical field experiments of point source release in Section 3. In Section 4 the model is applied to a natural ragweed field and compared with recent experimental results. An analysis of the deposition patterns is presented in Section 5 and the main results are discussed in Section 6.

\section{Model description}

The LES code used in this work solves the three-dimensional filtered momentum equations in rotational form. The numerical discretization combines a fully dealiased pseudo-spectral approach in the horizontal directions with a second-order centered finite-differences scheme in the vertical (described in more detail in Albertson \& Parlange, 1999; Kumar, Kleissl, Parlange, \& Meneveau, 2006). The fully explicit second-order Adams-Bashforth scheme is used for time integration. The dynamic subgridscale (SGS) model (Germano, Piomelli, Moin, \& Cabot, 1991) is used following the Lagrangian scale-dependent implementation of Bou-Zeid, Meneveau, and Parlange (2005).

Boundary conditions for the momentum equations are imposed using a wall layer model (Piomelli \& Balaras, 2002). We follow the approach of Moeng (1984), using the more recent implementation of Bou-Zeid et al. (2005), where the wall stress $\tau_{w}(x, y)$ is calculated based on the local test-filtered velocity components at the first grid point $z_{1}=d z / 2$. For the simulations of the ragweed field presented in Section 4, the canopy cannot be resolved in the grid used and its effect on the wind field is parameterized through the boundary condition. Several tests performed (not presented here) show that the velocity field in the first vertical grid point cannot be reproduced if a single length scale (the surface roughness $z_{0}(x, y)$ ) is used to represent the canopy. A better representation of the wind field close to the canopy is obtained if an additional length scale is introduced, the displacement height $d_{0}(x, y)$ (e.g. Kaimal \& Finnigan, 1994). The wall model is thus given by

$$
\tau_{w}(x, y)=-\left[\frac{\kappa}{\log \left(\frac{z_{1}-d_{0}(x, y)}{z_{0}(x, y)}\right)}\right]^{2}\left(\hat{\tilde{u}}_{1}^{2}\left(x, y, z_{1}\right)+\hat{\tilde{u}}_{2}^{2}\left(x, y, z_{1}\right)\right),
$$

where $\kappa=0.4$ is the vón Kárman constant, $u_{1}$ and $u_{2}$ are the horizontal components of velocity (and $u_{3}$ is the vertical component), a tilde indicates a quantity represented at grid scale $(\Delta)$ and a caret represents a test-filtered quantity at a larger scale $(2 \Delta)$. The total stress is then partitioned into streamwise and cross-stream components as in Bou-Zeid et al. (2005).

\subsection{Pollen conservation equation and SGS model}

An Eulerian approach is used in order to simulate pollen dispersal. A continuous pollen concentration field is assumed and represented by $C(x, y, z ; t)$. The evolution of the concentration field is obtained from the conservation of pollen mass, represented 
by the advection-diffusion equation with a term representing the gravitational settling. Neglecting the small effect of molecular diffusion, the final form of the equation for the resolved pollen concentration reads

$$
\frac{\partial \widetilde{C}}{\partial t}+\left(\widetilde{\mathbf{u}}-w_{s} \mathbf{e}_{3}\right) \cdot \nabla \widetilde{C}=-\nabla \cdot \pi^{C}+Q_{s r c}
$$

In Eq. (2) $w_{s}$ is the settling velocity of pollen grains (assumed to be constant and equal to the terminal velocity in a still fluid), $Q_{s r c}$ is a pollen source term, and $\pi^{C}$ is the SGS pollen flux defined as

$$
\pi^{C} \equiv \widetilde{\mathbf{u} C}-\widetilde{\mathbf{u}} \widetilde{C}
$$

The SGS pollen flux is parameterized using a flux-gradient model (analogous to the Smagorinsky model for the SGS stress tensor)

$$
\pi_{\text {model }}^{C}=-\frac{v_{s g s}}{S c_{s g s}} \nabla \widetilde{C}
$$

where $v_{s g s}$ is the SGS viscosity obtained using the Lagrangian scale-dependent dynamic model and $S c_{s g s}$ is a prescribed constant SGS Schmidt number (the value adopted here is $S c_{s g s}=0.4$ ).

\subsection{Pollen boundary conditions}

The boundary condition for pollen concentration at the surface is extremely important since in the proposed approach it is used to represent both the pollen emission and the ground deposition. This problem is common to other applications such as snow transport (e.g. Lehning, Löwe, Ryser, \& Raderschall, 2008) and sand transport (e.g. Shao, 2001), where erosion/deposition processes have to be modeled through the boundary condition.

The most common way to specify boundary conditions for the velocity and other scalar fields (usually temperature) in LES of the atmospheric boundary layer is by using the Monin-Obukhov similarity theory to prescribe the wall functions (e.g. Moeng, 1984). We follow the same approach to specify the lower boundary conditions for pollen concentration. Chamberlain (1967) derived an equilibrium profile for concentration of suspended particles under neutral atmospheric stratification assuming the eddy diffusivity for particles to be the same as the one for momentum (this result was independently derived much later by Kind, 1992). Chamecki, van Hout, Meneveau, and Parlange (2007) generalized Chamberlain's results by including the effects of thermal stratification on the vertical profile. They also included the average effect of the vegetation on the profile by incorporating the displacement height concept in the eddy diffusivity formulation and allowed the particle eddy diffusivity to be different from that of momentum. The applicability of the resulting expressions was verified against experimental measured profiles of corn pollen. Here only the expression for neutral atmospheric stability given by

$$
\frac{C}{C_{r}}=\left(\frac{\Phi}{C_{r} w_{s}}+1\right)\left(\frac{z-d_{0}}{z_{r}-d_{0}}\right)^{-\alpha}-\frac{\Phi}{C_{r} w_{s}}, \quad \alpha=\frac{S c_{T} w_{s}}{\kappa u_{*}}
$$

is used.

In Eq. (5), $C_{r}$ is the pollen concentration at the reference level $z_{r}, u_{*}$ is the friction velocity, and $\Phi$ is the pollen surface flux. The parameter $\alpha$ represents the ratio between settling velocity and a turbulent diffusive velocity at the boundary. Note that in principle the turbulent Schmidt number $S c_{T}$ does not have to be equal to the $S G S S c h m i d t$ number $S c_{s g s}$. In fact, we use $S c_{T}=0.95$, which yields the generally accepted form of Monin-Obukhov similarity function for scalars (e.g. Högström, 1988) if the limit of $w_{s} \rightarrow 0$ is adopted. Eq. (5) can be rearranged and expressed in terms of resolved quantities to yield the required expression for the SGS pollen flux at the surface $\left(\Phi_{\text {sgs }}\right)$ :

$$
\Phi_{s g s}(x, y)=-w_{s} \frac{\widetilde{C}\left(x, y, z_{1}\right)-C_{r}\left(\frac{z_{1}-d_{0}(x, y)}{z_{r}(x, y)-d_{0}(x, y)}\right)^{-\alpha}}{1-\left(\frac{z_{1}-d_{0}(x, y)}{z_{r}(x, y)-d_{0}(x, y)}\right)^{-\alpha}} .
$$

The SGS pollen flux $\left(\Phi_{s g s}\right)$ can then be determined once the reference value $C_{r}$ at $z_{r}$ is specified. The proposed approach is to model ground deposition by simply specifying $C_{r}=0$ at $z_{r}=z_{0, c}$, where $z_{0, c}$ is the surface roughness for pollen concentration and represents the height of the equivalent sink of pollen (note that this is equivalent to assume that once pollen grains are deposited, they are not available for re-suspension). In this case, Eq. (6) reduces to

$$
\Phi_{s g s}^{\text {dep. }}(x, y)=-w_{s} \widetilde{C}\left(x, y, z_{1}\right)\left(\frac{z_{1}^{\alpha}}{z_{1}^{\alpha}-z_{0, c}^{\alpha}}\right) .
$$

Eq. (7) was first obtained by Chamberlain (1967), who also noted that for rough surfaces $z_{0, c}=z_{0}$ yields a good approximation. The specification of field sources using Eq. (6) is accomplished by imposing measured concentration values $\left(C_{r}\right)$ above the source field and will be discussed in detail in Section 4 . The upper boundary condition is a no-flux condition and the height of the domain is chosen large enough such that this is a good approximation. 
As discussed in detail by Chamecki et al. (2007), the flux calculated from Eq. (6) is composed by the superposition of the gravitational settling and the turbulent diffusive vertical fluxes. Although the former acts always as a sink in the simulation, the second can be either a source or a sink, depending on the concentration gradient. It is of interest here to separate the source and deposition (sink) components of the total flux. Eq. (6) can be rearranged as

$$
\Phi_{s g s}(x, y)=\Phi_{s g s}^{s r c}(x, y)+\Phi_{s g s}^{d e p}(x, y),
$$

where

$$
\Phi_{s g s}^{s r c}(x, y)=w_{s} C_{r}\left(\frac{z_{1}-d_{0}(x, y)}{z_{r}(x, y)-d_{0}(x, y)}\right)^{-\alpha}\left[1-\left(\frac{z_{1}-d_{0}(x, y)}{z_{r}(x, y)-d_{0}(x, y)}\right)^{-\alpha}\right]^{-1}
$$

and

$$
\Phi_{\text {sgs }}^{\text {dep }}(x, y)=-w_{s} \widetilde{C}\left(x, y, z_{1}\right)\left[1-\left(\frac{z_{1}-d_{0}(x, y)}{z_{r}(x, y)-d_{0}(x, y)}\right)^{-\alpha}\right]^{-1} .
$$

Note that Eq. (9) is obtained by replacing $\widetilde{C}\left(x, y, z_{1}\right)=0$ into the original Eq. (6) and can be interpreted as the total amount of pollen emitted from the field. On the other hand, Eq. (10) is obtained by replacing $C_{r}=0$ which is the approach used to parameterize the deposition flux. Using this decomposition, the two separate parts of the net flux can be integrated during the simulation and the total net flux can be further separated into the total emission and deposition parts.

The use of Eq. (6) to model the pollen sources and sinks has some similarities with the recent model proposed for snow transport by Lehning et al. (2008). In their model, the surface flux is specified by superimposing the turbulent diffusive flux to the gravitational settling and then approximating the vertical concentration gradient in the former using a first-order expansion. Instead of using such an approximation, we use the analytical solution (5) to the same flux equation.

\subsection{Numerical discretization}

The pollen sources (both the point and field sources) are fairly localized in space, generating strongly nonhomogeneous concentration fields. For this reason, the pseudo-spectral approach, which is used to discretize the velocity field is not a good option for the pollen concentration field. It can generate unphysical behavior such as spatial oscillations and negative values of pollen concentration. Therefore, we discretize the pollen conservation equation using a finite-volume approach. The advection term is discretized using the bounded third-order upwind interpolation scheme SMART proposed by Gaskell and Lau (1988). This scheme prevents by construction occurrence of negative concentrations and was successfully used by Xie, Hayden, Voke, and Robins (2004) to simulate scalar diffusion from point sources. The usual second-order centered scheme is used for the turbulent diffusion term. Coupling between the finite-volume discretization for the pollen concentration with the pseudospectral discretization for the momentum equations requires interpolation of the velocity field from the spectral nodes to the finite-volume surfaces. The conservative interpolation scheme proposed by Chamecki, Meneveau, and Parlange (2008) is used to ensure that conservation of mass is satisfied to high precision on the local finite-volume discretization.

\section{Point source simulations}

In order to validate the proposed numerical model, simulations of point source release reproducing field experiments were carried out. By eliminating all the uncertainty of the pollen release by real plants, these cases provide adequate tests to assess the modeling of turbulent transport, SGS modeling, gravitational settling and deposition. Two different field experiments spanning a wide range of $w_{s} / u_{*}$ were simulated and the results are presented below.

\subsection{The Suffield experiment}

In the literature, there are a few experimental reports on point source releases in the atmospheric boundary layer. However, the Suffield experiment has been used as a test case to validate numerical (Wilson, 2000) and analytical (Bouvet \& Wilson, 2006) models of heavy particle dispersion. The experiments at the Suffield Research Station (Canada) consisted of releasing glass microspheres continuously from an elevated point source. Ground deposition was measured by counting spheres on flat-plate adhesive surfaces distributed in concentric arcs around the source. The main results were reported by Hage (1961) and Walker (1965). Using the mean velocity and temperature profiles, Wilson (2000) estimated values for friction velocity ( $\left.u_{*}\right)$, Obukhov length $(L)$ and surface roughness $\left(z_{0}\right)$ for each of the 12 runs. The run labeled 5 by Hage (1961) and C by Walker (1965) is the closest one to neutral stability. This is a 60 -min release from a height $h=15 \mathrm{~m}$. The average particle diameter is $d=107 \mu \mathrm{m}$ and the settling velocity in still fluid $w_{s}=0.58 \mathrm{~m} \mathrm{~s}^{-1}$. The total mass of spheres released was $Q_{t}=427.5 \mathrm{~g}$, corresponding to a constant emission rate $Q=0.11875 \mathrm{~g} \mathrm{~s}^{-1}$. Wilson's estimates for this run are $u_{*}=0.44 \mathrm{~m} \mathrm{~s}^{-1}, z_{0}=0.025 \mathrm{~m}$ and $L=341 \mathrm{~m}$ (neutral stability is assumed in the simulation). The point source is discretized using the minimum possible number of finite volumes to mimic a source at $z=15 \mathrm{~m}$. The source strength used is given by $Q_{s r c}=Q / V_{s r c}$ where $Q$ is the emission rate from the experimental data 
Table 1

Numerical grids for simulation of Suffieldpoint source

\begin{tabular}{|c|c|c|c|c|}
\hline Parameter (unit) & Symbol & High & Intermediate & Coarse \\
\hline Grid points & $N_{x} \times N_{y} \times N_{z}$ & $128 \times 64 \times 96$ & $64 \times 32 \times 48$ & $32 \times 16 \times 24$ \\
\hline Domain length (m) & $L x$ & 640 & 640 & 640 \\
\hline Domain width (m) & Ly & 320 & 320 & 320 \\
\hline Domain height (m) & $H$ & 96 & 96 & 96 \\
\hline Horizontal grid spacing $(\mathrm{m})$ & $\mathrm{d} x, \mathrm{~d} y$ & 5 & 10 & 20 \\
\hline Vertical grid spacing $(\mathrm{m})$ & $\mathrm{d} z$ & 1 & 2 & 4 \\
\hline Timestep (s) & $\mathrm{d} t$ & 0.03 & 0.05 & 0.05 \\
\hline Source location node & $(i, j, k)_{s r c}$ & $(20,32,15+16)$ & $(10,16,8)$ & $(5,8,4+5)$ \\
\hline Source location (m) & $(x, y, z)_{s r c}$ & $(97.5,157.5,15)$ & $(95,155,15)$ & $(90,150,15)$ \\
\hline Source volume $\left(\mathrm{m}^{3}\right)$ & $V_{s r c}$ & 50 & 200 & 3200 \\
\hline
\end{tabular}

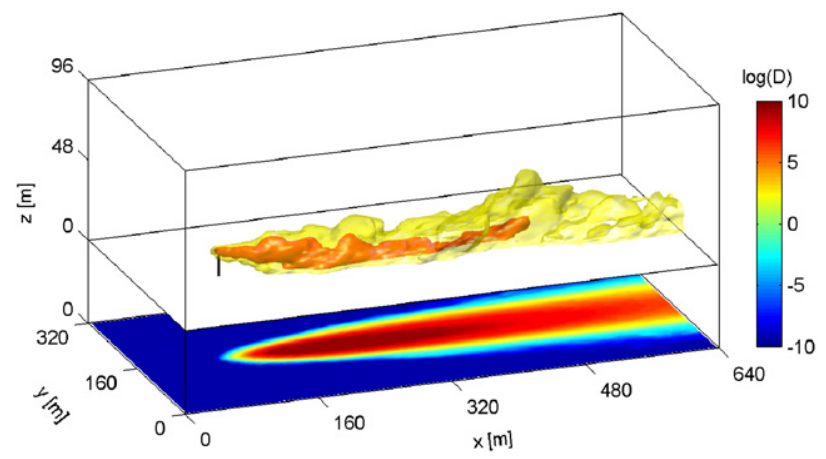

Fig. 1. Instantaneous particle concentration plumes for the Suffield case. Isosurface for $\widetilde{C}=1000 \mathrm{~g} \mathrm{~m}^{-3}$ (solid red surface) and $\widetilde{C}=1 \mathrm{~g} \mathrm{~m}^{-3}$ (transparent yellow surface). Also shown the final time-integrated ground deposition (plane is offset for better visualization).

a

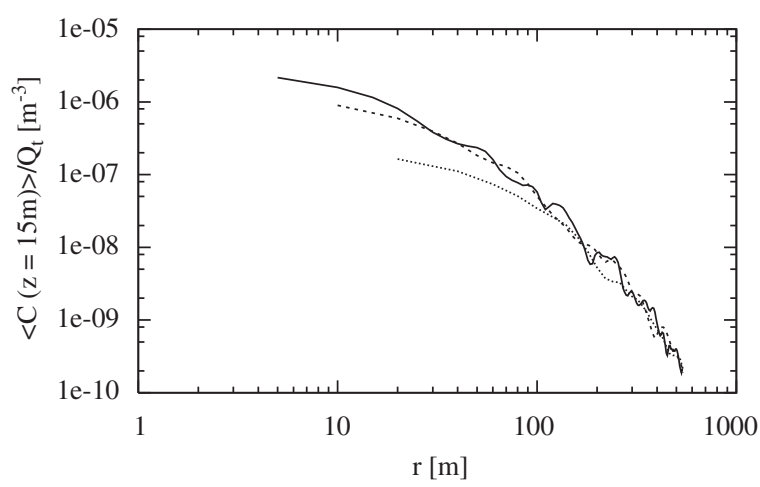

b

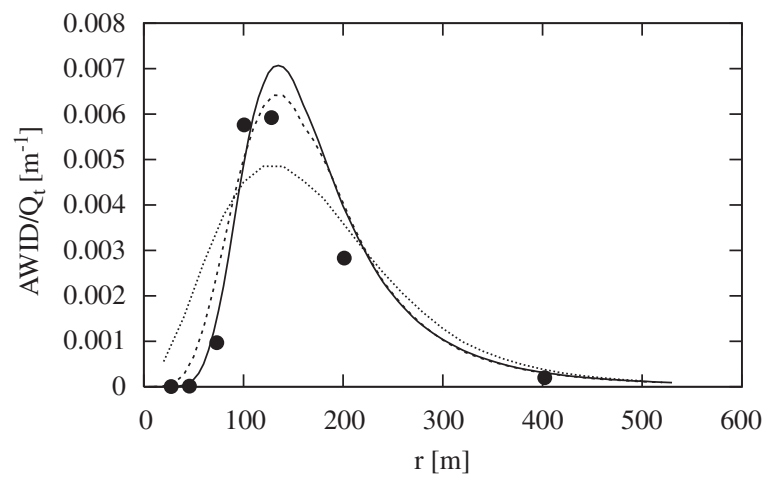

Fig. 2. (a) Mean centerline concentration at source height normalized by total amount of particles emitted and (b) normalized arcwise integrated deposition for the Suffield case. Experimental data (filled circles) and simulation results: high resolution (solid line), intermediate resolution (dashed line) and coarse resolution (dotted line).

and $V_{s r c}$ is the volume used to represent the source in the finite-volume mesh. Three simulations are carried out with progressive mesh refinement (see Table 1 ).

An instantaneous snapshot of the three-dimensional plumes (isosurfaces) for $\widetilde{C}=1000$ and $1 \mathrm{~g} \mathrm{~m}^{-3}$ are shown in Fig. 1 . The high concentration plume clearly illustrates the effect of the large settling velocity for the glass beads, as the plume has a strong inclination towards the ground surface. However, as can be seen in both plumes, strong enough turbulent fluctuations carry particles farther downwind. The two-dimensional ground deposition integrated during the entire simulation is also shown, the region of larger deposition corresponding to the region where the high concentration plume touches the ground.

The centerline airborne concentration at the height of the source $z=15 \mathrm{~m}$ is shown in Fig. 2(a) as function of the distance from the source $r$ (no experimental data available). Despite differences close to the source, all the simulations agree well in the far field. This is an encouraging result, implying that if only the far field is of interest, grid resolution is not a critical factor.

The ground deposition data reported by Hage (1961) and Walker (1965) can be used to validate the dispersion from the LES. Measurements were made along 8 arcs centered at the source. Only the arcwise integrated deposition (AWID) and the cross-arc 


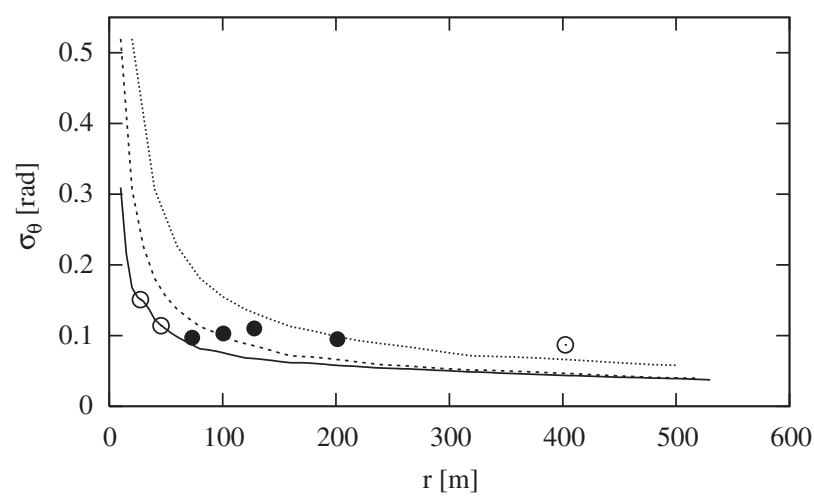

Fig. 3. Cross-arc standard deviations for the Suffield case. Experimental data (filled circles), unreliable measured data (open circles) and simulation results (see legend in Fig. 2).

a

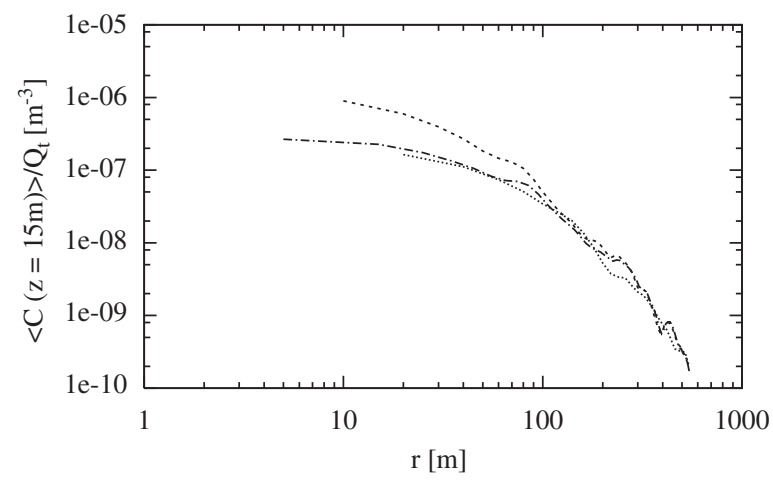

b

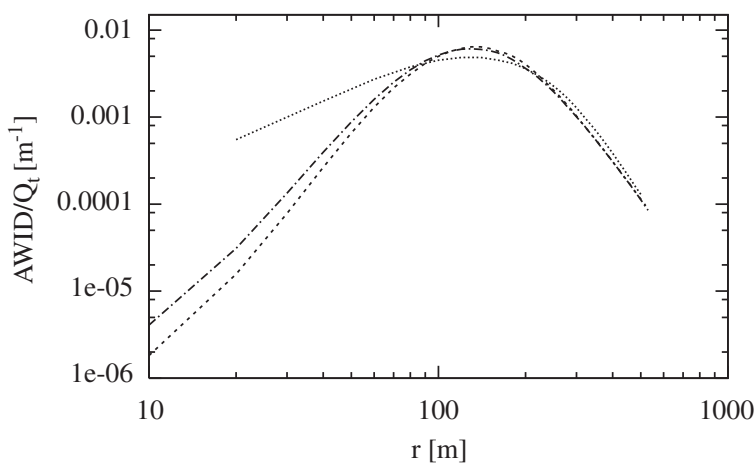

Fig. 4. Source discretization tests for the Suffield case: (a) Mean normalized centerline concentration at source height and (b) normalized arcwise integrated deposition. Intermediate resolution (dashed line), coarse resolution (dotted line) and test simulation (dash-dotted line).

standard deviation $\left(\sigma_{\theta}\right)$ are reported. In Fig. 2(b) the AWID normalized by the total emission is presented as a function of radius. The general agreement is very good for the intermediate and high resolution simulations, but quite poor for the coarser one. The peak in deposition is correctly predicted (both its position and intensity) and the width is also well represented. Very good agreement is obtained for the near field in the high resolution case. Again, it is clear that even the coarser resolution is capable of predicting the far field deposition with good accuracy.

The cross-arc standard deviation of the deposition is compared to the simulation in Fig. 3. Walker (1965) fitted a Gaussian distribution to each arc and reported the standard deviation values obtained. The comparison is fairly good but the simulations tend to underestimate the cross-arc spread in the far field.

The differences observed between the various resolutions in the near field can be either due to the lack of small scales in the coarser resolution which forces most of the dispersion into the SGS model or due to the impossibility of accurately representing the point source (note that in the coarse case the point source is represented by a volume of $3200 \mathrm{~m}^{3}$ ). In order to determine which is the main cause for the discrepancies, a fourth simulation was performed using the intermediate resolution but the coarser point source representation. The results for centerline concentration and ground deposition are presented in Fig. 4 . The differences observed between the intermediate and the coarser resolutions at source height are clearly an effect of the point source discretization, as evidenced by the overlap between this new simulation and the coarse resolution simulation. However, the differences observed on the ground deposition are mostly caused by the resolution of the turbulence, since the overlap is now between the intermediate resolution and the new simulation. The conclusion is that finer resolutions are indeed required if one is interested in accurately predicting the near field deposition.

\subsection{The Brookhaven experiment}

A large number of field experiments of dispersion and deposition of pollen grains were performed in the 1960s in the Brookhaven National Laboratory. These experiments included point and area source releases of radioactive tracers and pollen grains of corn (Zea mays L.), timothy (Phleum prataense) and ragweed (Ambrosia) (e.g. Raynor, Hayes et al., 1972; Raynor, 
Table 2

Numerical grid for simulation of Ragweed pollen dispersal

\begin{tabular}{|c|c|c|c|}
\hline Parameter (unit) & Symbol & Brookhaven & Field \\
\hline Grid points & $N_{x} \times N_{y} \times N_{z}$ & $128 \times 64 \times 96$ & $150 \times 50 \times 50$ \\
\hline Domain length (m) & $L x$ & 128 & 1200 \\
\hline Domain width (m) & Ly & 64 & 600 \\
\hline Domain height (m) & $H$ & 19.2 & 150 \\
\hline Horizontal grid spacing (m) & $\mathrm{d} x, \mathrm{~d} y$ & 1 & 12 \\
\hline Vertical grid spacing (m) & $\mathrm{d} z$ & 0.2 & 3 \\
\hline Timestep (s) & $\mathrm{d} t$ & 0.0075 & 0.1 \\
\hline
\end{tabular}

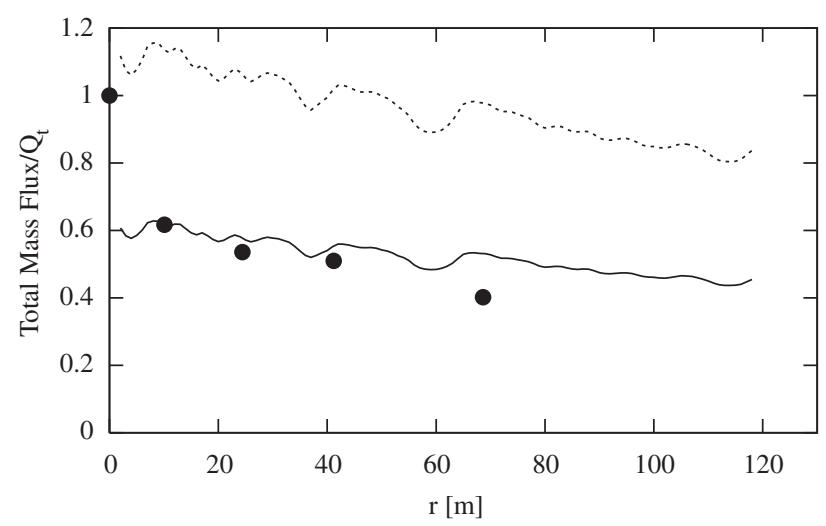

Fig. 5. Normalized total pollen mass flux for the Brookhaven case. Experimental data (filled circles), LES simulation (dashed line) and LES simulation with rescaled source strength (solid line).

Ogden et al., 1970, 1972). Among the three species, ragweed has the smallest pollen grains ( $d=20 \mu \mathrm{m})$ and provides a good test case for very small particles (as opposed to the fairly large glass beads from the previous section).

The data from the ragweed experiments are reported in detail by Raynor, Hayes, and Ogden (1970). Measurements of mean airborne pollen concentration and ground deposition were performed at five circles with different radius centered in the source. Airborne pollen concentrations were sampled at four different heights using slide-edge-cylinder samplers and ground deposition was measured using greased microscope slides.

From the 21 point source releases reported by Raynor, Hayes et al. (1970), the data from only seven cases were considered adequate to estimate mass fluxes. We chose the most neutral run (i.e. smallest deviation from fitted log-law profile) to simulate. This specific case (labeled 6025G) corresponds to a release of $4.75 \times 10^{8}$ grains in an interval $T=24 \mathrm{~min}$ from a height $h=1.5 \mathrm{~m}$. Friction velocity and surface roughness estimated from the fitted log-law were $u_{*}=0.35 \mathrm{~m} \mathrm{~s}^{-1}$ and $z_{0}=0.015 \mathrm{~m}$. The settling velocity of ragweed pollen grains reported by Raynor, Ogden et al. (1970) is $w_{s}=0.0156 \mathrm{~m} \mathrm{~s}^{-1}$. The grid resolution is chosen based on the intermediate resolution for the Suffield case (i.e. such that there are seven grid points between the source and the surface). The main characteristics of the grid are shown in Table 2.

Using the mean wind speed profile and the mean concentration plume, Raynor, Ogden et al. (1972) estimate the total number of pollen grains crossing the sampling circle with radius $10.1 \mathrm{~m}$. For the dataset used in this work, only $62 \%$ of the grains emitted cross this circle. This decrease seems too large for the small settling velocity of ragweed pollen grains. Furthermore, it does not seem consistent with the deposition pattern observed (see discussion of deposition below). Raynor, Ogden et al. (1972) attribute this discrepancy to uncertainties in the total emission and errors in the sampling and analysis procedures. The total mass flux, estimated based on mean velocities and concentrations from our simulation, is shown together with the data reported by Raynor, Ogden et al. (1970) in Fig. 5. Clearly the estimates of mass flux from the simulations also contradict the large decrease in mass flux between the source and the first measurement circle. In order to proceed, we rescale the source strength such that the mass flux of the simulation matches the experimental value at the $10.1 \mathrm{~m}$ circle (the source strength is reduced to $54 \%$ of its value). The result is also shown in the figure and a fairly good agreement with the data is evident.

Raynor, Ogden et al. (1970) also report the values of arcwise integrated airborne concentration (AWIC) at the height of the source for the five sampling rings. The simulation results are compared to the field data in Fig. 6(a). The overall agreement is quite good over the entire range of data. The decay of the AWID is shown in Fig. 6(b). Note that the values obtained from the simulation are much larger than those from the field experiment. However, as noted by Raynor, Ogden et al. (1972), deposition on smooth slides is usually much lower than on the rough field (mowed grass, in this case). Although a consistent approach to obtain realistic deposition from measurements using smooth slides is not available, it is possible to determine a correction for the simulated deposition using the theoretical deposition model (7). The correction factor is obtained by comparing the deposition model for the rough surface to a smooth wall and is found to be a function of the surface roughness, the viscous length scale 
a

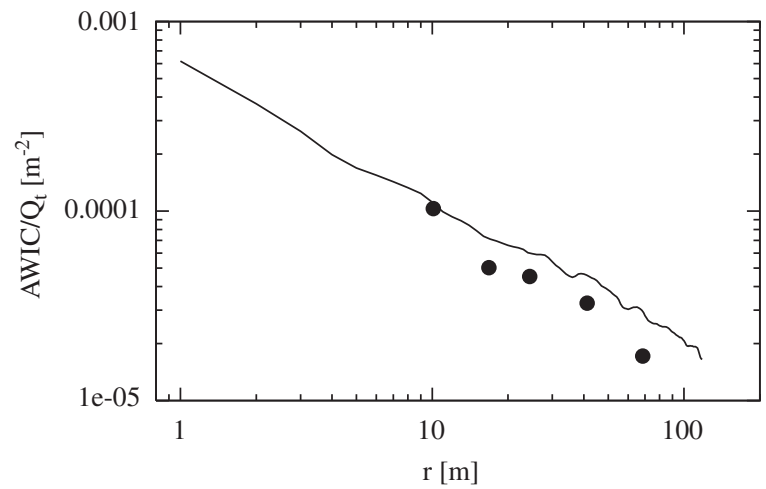

b

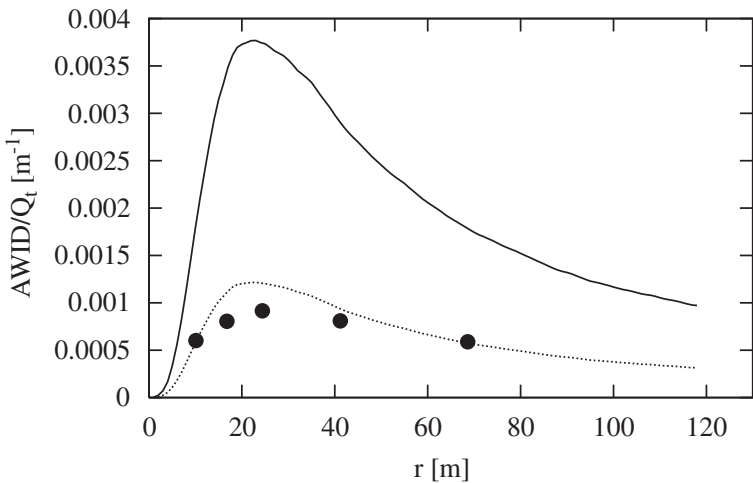

Fig. 6. (a) Normalized arcwise integrated mean concentration at source height and (b) normalized arcwise integrated deposition for the Brookhaven case. Experimental data (circles) and LES simulation (lines).

a

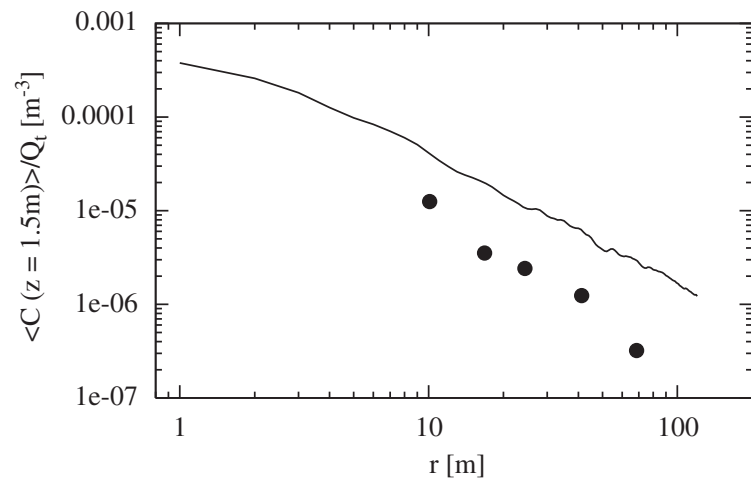

b

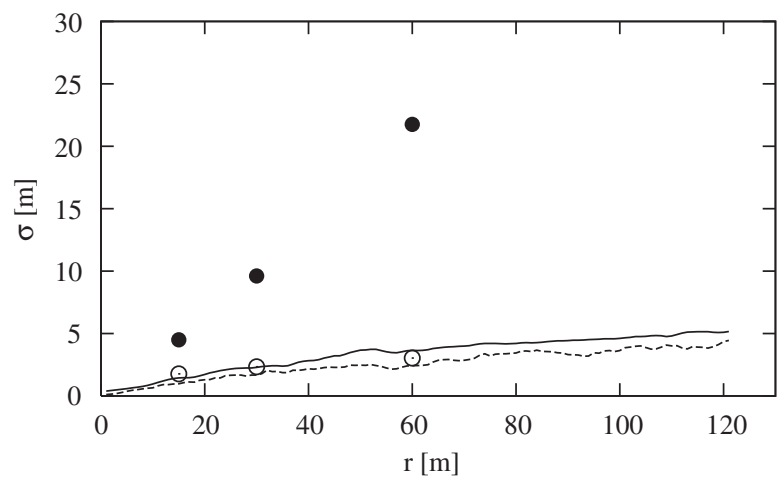

Fig. 7. (a) Normalized mean centerline concentration at source height and (b) growth of the pollen plume for the Brookhaven case. Experimental data (circles) and LES simulation (lines). In panel (b), the plume width $\sigma_{y}$ is represented by filled circles and solid line, and the plume height $\sigma_{z}$ by empty circles and dashed line.

and the height of the first grid point. The details are presented in the Appendix; for the present case the correction factor that must be applied to the simulation result is obtained to be $\gamma=3.10$. If this value is used to estimate what the deposition from the simulation on smooth slides would be, the agreement is very good.

Despite the good agreement in the arcwise integrated concentration and deposition, there are some difficulties reproducing the centerline counterparts. Centerline concentration at source height is shown in Fig. 7(a). Clearly the simulation overestimates the values and predicts a slower decay. The same conclusion is valid for the centerline deposition (not shown).

The main reason for the discrepancy in the centerline quantities becomes evident when one looks at the plume growth. In Fig. 7(b) the values of the plume width and plume height (estimated by fitting Gaussian curves to the average concentration field) are compared to the experimental data. Although the vertical spread is fairly well reproduced, the horizontal spread is underestimated by about a factor of 5. Raynor, Ogden et al. (1970) also noted that the horizontal spread from the experimental data seemed a bit too large. However, it seems clear that even if the field data are slightly overestimated, the simulation is not able to reproduce the horizontal growth of the plume.

A second simulation using a coarser grid is used to verify whether convergence issues might play an important role in the horizontal spread predicted by the model. The results indicate that this is not the case (not shown here). Although the simulation with the finer grid has a better prediction of $\sigma_{z}$, this is not the case for $\sigma_{y}$. The coarser simulation in fact has a slightly larger horizontal spread.

The discrepancy in the horizontal spread of the plume is likely to be caused by the absence of mesoscale variations in wind directions (i.e. meandering winds). The importance of these motions in predicting horizontal dispersal under light wind conditions has been documented by Anfossi et al. (2006). Recent work by Vickers, Mahrt, and Belusic (2007) extends the importance of meandering winds for dispersion under all atmospheric conditions and shows that meandering (and not turbulence) dominates the $1 \mathrm{~h}$ averaged horizontal dispersion. In the present numerical model, these meandering motions could be introduced by and 
unsteady direction in the mean pressure gradient that drives the simulation or by incorporating realistic boundary conditions from mesoscale models. However, since these effects are likely to be less important in the case of a large area source, we proceed with the current approach and leave the investigation of alternative possibilities for future research.

\section{Ragweed field simulations}

\subsection{Experimental data}

In this section the numerical code is applied to more realistic conditions where pollen is emitted from a natural area source. A field experiment was carried out during the months of September and October of 2006 in a fairly flat field near Upper Marlboro, MD. The $245 \mathrm{~m} \times 245 \mathrm{~m}$ occupied homogeneously by common ragweed (Ambrosia artemisiifolia). The average height of the plants was $0.69 \mathrm{~m}$ and the estimated density was 89 plants per square meters. The canopy height was increased considerably by a homogeneous distribution of mare's tail (Conyza canadensis) with a density of 6 plants per square meters. The field experiment consisted of simultaneous measurements of meteorological and turbulent quantities and pollen release, airborne concentration and ground deposition (see Martin, Chamecki, Brush, Meneveau, \& Parlange, 2008, for more details). Only the part of the data collected relevant for this work is described in the sequence.

Two different setups were employed to study vertical and horizontal pollen dispersal. For the first setup, intended to study vertical dispersal, the field was kept undisturbed and the only data used here are the profiles of mean wind speed from three cup anemometers placed at 2, 4 and $6 \mathrm{~m}$ above the ground (these data are used to estimate the characteristic surface roughness and the corresponding displacement height for the field).

We base our simulations on the second setup, which was designed to study horizontal dispersion and ground deposition. The field was mowed, leaving only a north-south stripe ragweed patch of $48 \mathrm{~m} \times 245 \mathrm{~m}$. A 6 m tower with meteorologic instrumentation was placed upwind of the ragweed patch as shown in the sketch presented in Fig. 8. Two CSAT3 sonic anemometers and a Krypton Hygrometer positioned $3 \mathrm{~m}$ above the ground were used to measure momentum, heat and water vapor turbulent fluxes. Mean wind speed and direction, temperature, relative humidity and net radiation were also measured but are not used here. Measurements of airborne pollen concentrations were performed using six Rotorod (Multidata) rotating impact samplers. Five samplers were placed at the downwind (east) edge of the field to characterize the pollen source: one at the southern station $(z=2 \mathrm{~m})$, three at the center station $(z=2,2.5$ and $3 \mathrm{~m})$ and one at the northern station $(z=2 \mathrm{~m})$. The last sampler was placed upwind to characterize pollen concentration from other nearby possible sources (concentrations on this sampler were insignificant during the entire experiment). Finally two lines of greased microscope slides were positioned downwind to measure ground pollen deposition as a function of distance from the field (placed at 4, 8, 12, 16, 20, 24, 28, 36, 44 and $52 \mathrm{~m}$ from the edge of the field). From all data available for the second setup, September 29th was chosen to be simulated, because the wind direction was steady from the northwest all day. Although this was a bit late in the season, significant amounts of ragweed pollen were being emitted from the field.

\subsection{Simulation setup}

The simulation domain chosen is larger than the experimental site $(L x \times L y \times H=1200 \mathrm{~m} \times 600 \mathrm{~m} \times 150 \mathrm{~m})$ with a grid of $100 \times 50 \times 50$ points. The grid resolution $(\mathrm{d} x \times \mathrm{d} y \times \mathrm{d} z=12 \mathrm{~m} \times 12 \mathrm{~m} \times 3 \mathrm{~m})$ is fairly coarse compared to the point source simulations in the previous section. The coarse resolution and the large domain are intentionally designed: ragweed pollen has a small settling velocity and spreads far from the source. If the main purpose is to simulate relevant length scales for ragweed pollen dispersion, coarse resolutions are unavoidable. A comparison of domain sizes and resolutions between the point source and the field simulations is shown in Table 2.

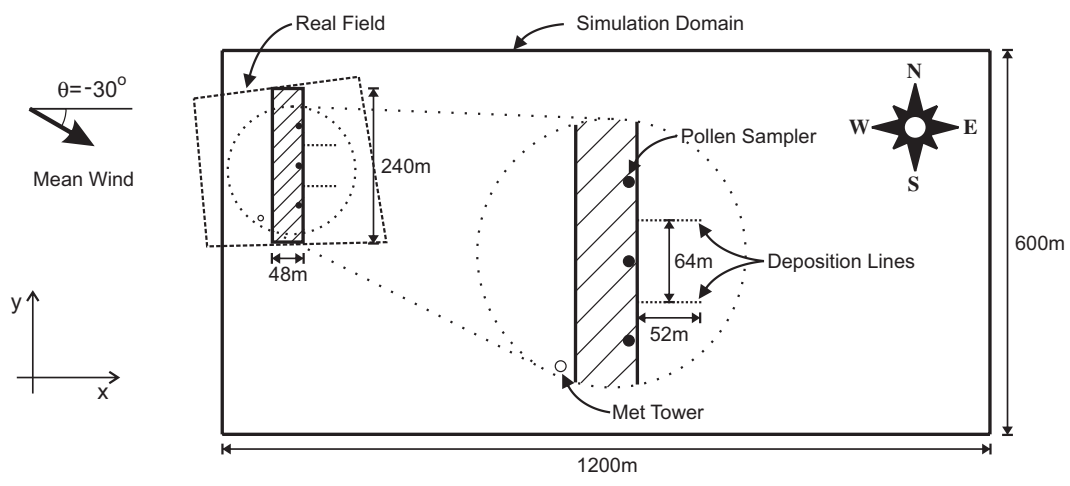

Fig. 8. Sketch of the domain for the ragweed field simulations. 
a

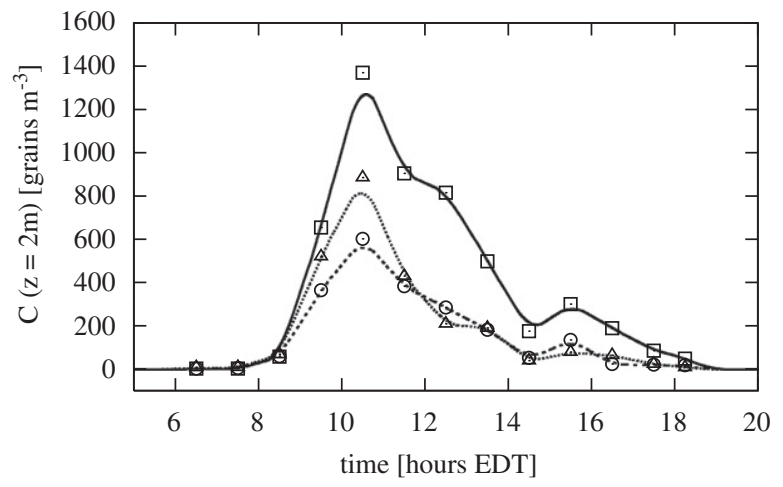

b

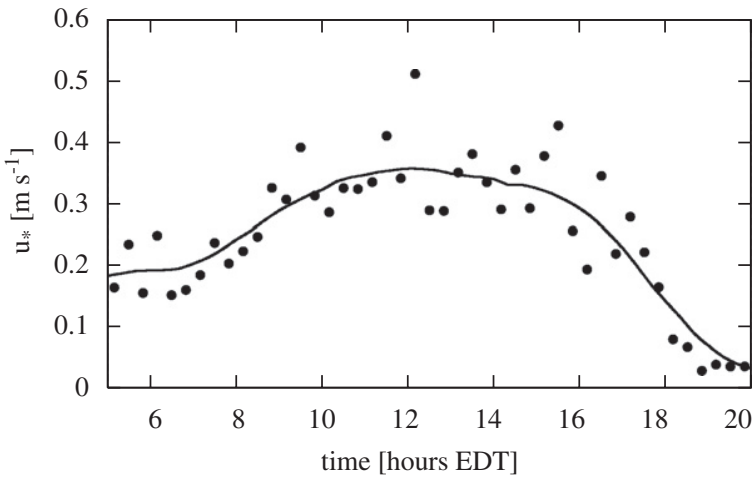

Fig. 9. Input data for ragweed field simulation (a) pollen concentration at $z=2 \mathrm{~m}$ and (b) friction velocity. Experimental data (filled circles) and smoothed boundary condition for simulation (solid line).

Over the region where the ragweed was mowed, the typical height was about $0.1 \mathrm{~m}$ and the values $d_{0}=0$ and $z_{0}=0.01 \mathrm{~m}$ are used in the simulation. The determination of both parameters over the ragweed patch is more complicated. We use velocity profiles obtained using the three cup anemometers from the first field setup (the homogeneous field) to estimate the appropriate values. The data were averaged over $1 \mathrm{~h}$ periods and only nearly neutral periods $(L \geqslant 200 \mathrm{~m})$ with adequate wind speeds $\left(\bar{u}(z=2 \mathrm{~m})>0.5 \mathrm{~m} \mathrm{~s}^{-1}\right)$ were used. The velocity profiles were normalized by the corresponding friction velocity (from the sonic anemometer) and then averaged. The values $z_{0}=0.36 \mathrm{~m}$ and $d_{0}=0.74 \mathrm{~m}$ minimize the mean square error between the data and the theoretical profile.

In order to complete the simulation setup, the diurnal variation of the turbulent conditions has to be reproduced and the pollen source has to be specified. The time evolution of the friction velocity and the pollen concentration at $z=2 \mathrm{~m}$ from the experiment are used for this purpose. Their diurnal variation is shown in Fig. 9, together with the smoothed curves which are used in the simulation as described in the sequence.

The model used here is driven by a mean pressure gradient, $\nabla p$, which, in steady state, is balanced by the surface friction so that $(1 / \rho)|\nabla p|=u_{*}^{2} / H$. In order to reproduce the mean wind direction (as illustrated in Fig. 8), the total pressure gradient imposed is decomposed such that $\nabla p /|\nabla p|=\cos \left(-30^{\circ}\right) \mathbf{i}+\sin \left(-30^{\circ}\right) \mathbf{j}$, where $\mathbf{i}$ and $\mathbf{j}$ are unit vectors in the $x$ and $y$ directions, respectively. The last step is to find the time-dependent pressure gradient magnitude $F_{p}(t)=(H / \rho)|\nabla p|$ that yields the measured time evolution of friction velocity $u_{*}(t)$ (i.e. the smooth curve in Fig. $\left.9(\mathrm{~b})\right)$ ). There are two main problems if $F_{p}(t)=\left[u_{*}(t)\right]^{2}$ is used: (1) the heterogeneous surface characteristics will cause an uneven distribution of the wall stress, yielding smaller values of $u_{*}$ over the smooth part (where measurements of friction velocity were made) and larger values over the ragweed patch; (2) there is a response time lag between the change in pressure forcing and the change in the friction velocity.

Test simulations with coarser resolution were used to investigate both issues. For the area distribution and specific values of surface roughness in the simulation domain used, numerical simulations with a steady pressure gradient showed that the value of the friction velocity on the smooth patch was approximately $10 \%$ smaller than the corresponding imposed pressure gradient. So the solution to the first problem is accomplished by setting $F_{p}(t)=\left[1.1 u_{*}(t)\right]^{2}$.

In order to study the second problem, a coarser simulation with a homogeneous domain (i.e. no ragweed patch) and $F_{p}(t)=$ $\left[u_{*}(t)\right]^{2}$ was carried out and the results are shown in Fig. 10(a). The friction velocity obtained from the simulation $u_{*}^{L E S}(t)$ displays two main deviations from the imposed pressure gradient: there is a clear time lag between the two curves and the final part of the curve decays more slowly. The time shift $\tau$ that yields the minimum mean squared error between the two curves is $\tau=5180 \mathrm{~s}$. Results for a new simulation with $F_{p}(t)=\left[u_{*}(t+\tau)\right]^{2}$ are shown in Fig. 10(b). The agreement between experimental and simulated friction velocities is very good until 17:00 h, when the experimental curve decays much faster. An analysis of the meteorological data indicates that this is exactly the time when the surface heat flux changes sign and stratification effects suppress the turbulent fluctuations. For the present purposes, the results presented in Fig. 10(b) represent a good approximation since almost no pollen remains airborne at this time (see Fig. 9(a)).

The final step is to specify the pollen source. The three curves in Fig. 9(a) suggest a very heterogeneous pollen emission. Since only three points are available to characterize the spatial distribution of $C_{r}(x, y, t)$ a few assumptions are needed. The source is assumed to be homogeneous in $x$ so that only $C_{r}(y, t)$ has to be determined. Linear interpolation in the $y$ direction is used between the points of measurement and constant concentration is assumed beyond the outer points.

\subsection{Simulation validation}

Three-dimensional fields were output every $10 \mathrm{~s}$ and further averaged in blocks of $5 \mathrm{~min}$. Values of the friction velocity at the point where the meteorological tower was located are compared to the 20-min averaged measurements in Fig. 11(a). Except 
a

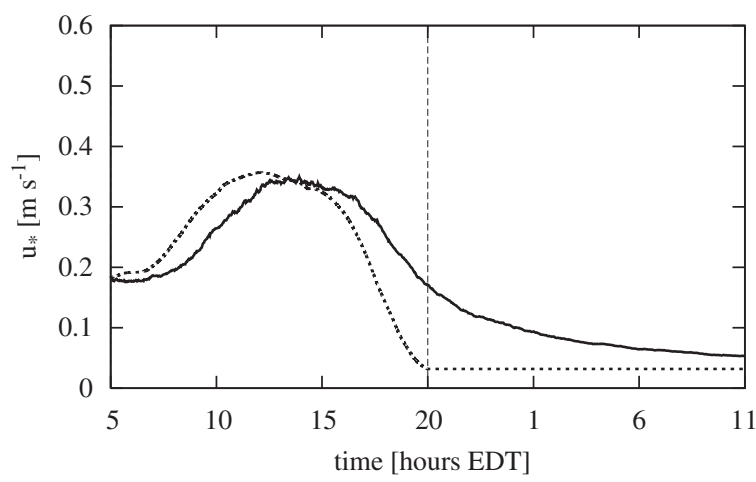

b

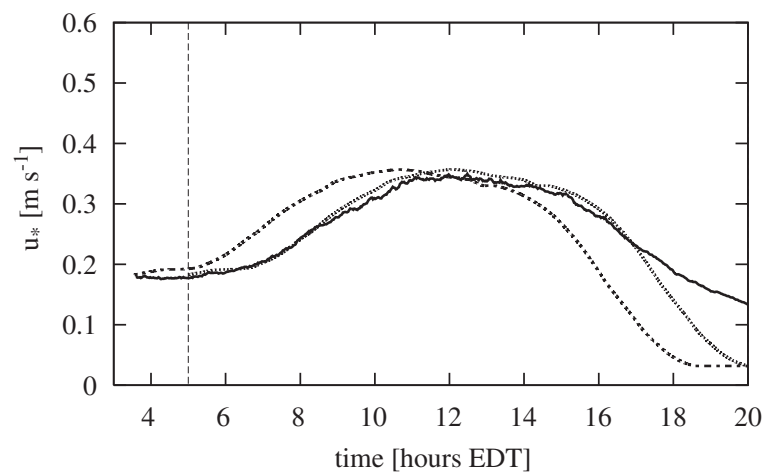

Fig. 10. Relation between imposed $F_{p}(t)$ and simulated $u_{*}^{L E S}(t)$. (a) The imposed $F_{p}(t)$ coincides with the experimental $u_{*}(t)$ (dashed line) but there is a lag in the simulated $u_{*}^{L E S}(t)$ (solid line). (b) The imposed $F_{p}(t)$ (dashed line) is shifted with respect to the experimental $u_{*}(t)$ (dotted line) and a good agreement is obtained from the simulated $u_{*}^{L E S}(t)$ (solid line).

a

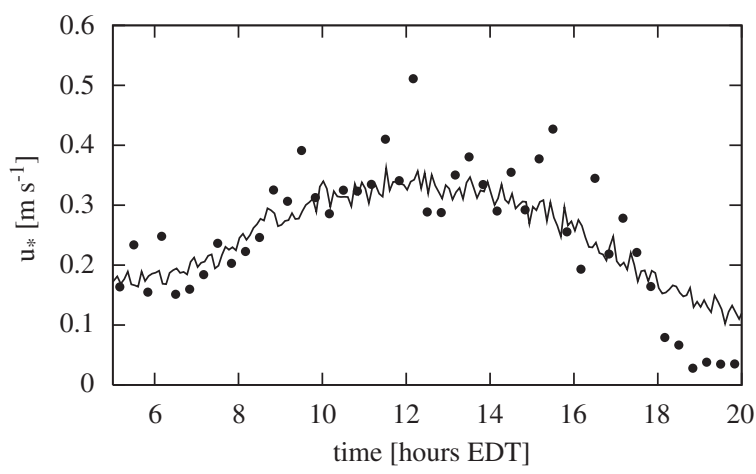

b

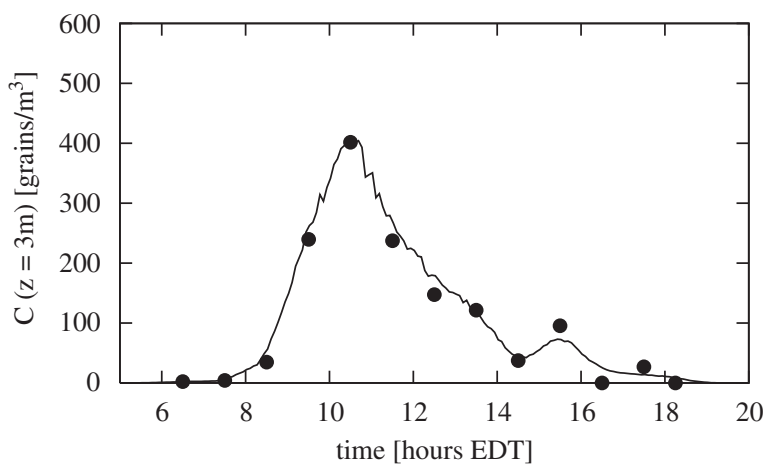

Fig. 11. (a) Friction velocity and (b) pollen concentration $3 \mathrm{~m}$ above the ground. Experimental data (filled circles) and LES simulation (solid line).

for the interval between 18:00 and 20:00, the agreement is very good. Pollen concentration at $z=3 \mathrm{~m}$ for the center sampler is compared to the simulated concentration at the same point in Fig. 11(b). The good agreement should be considered more a validation of the boundary condition model than for the entire model, since the concentration at this location is dominated by the value imposed through the boundary condition underneath.

Finally, a comparison between ground deposition measurements and simulated deposition is shown in Fig. 12 for the two deposition lines. As for the Brookhaven simulations in Section 3, curves representing the simulated deposition on smooth slides are also presented (however, in this case, the correction is based on $u_{*}(x, y, t)$ and not on the average value as in the previous case). The agreement is good for the north line shown in Fig. 12(a) and not as good for the south line in Fig. 12(b). In particular for the latter, the simulation seems to underestimate the deposition very close to the field $(x<6 \mathrm{~m})$ and overestimate it farther away. The agreement is not as good as for the point sources, but still remarkable given all the uncertainties in the specification of the source. The pollen deposition inside the entire computational domain is shown in Fig. 13. Effects of the mean wind direction and heterogeneity of the pollen source have a strong effect on the deposition pattern.

\section{Analysis of pollen deposition}

An accurate estimate of the total amount of pollen emitted from a field, and its partition in deposition inside and outside the source field, plays an important role in determining amount and purity of seed production in crops and in assessing risks of cross-pollination from genetically modified crops (e.g. Aylor, 2005). It is also an important factor in determining patterns of gene flow between plant populations, a key effect in plant evolutionary and conservation biology (e.g. Ellstrand, 1992). However, total pollen emission from a field and the deposition inside the source field itself are very difficult to measure experimentally. Aylor and Flesch (2001) proposed a method to estimate the total emission based on measurements of airborne concentrations coupled to a two-dimensional Lagrangian stochastic model. 
a

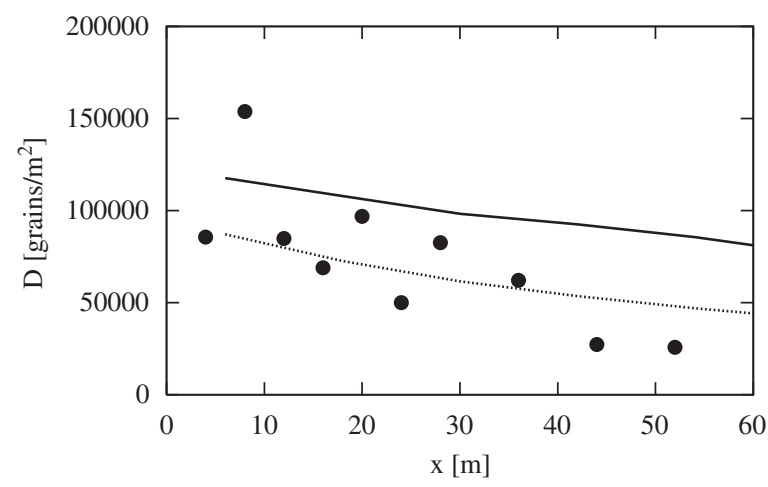

b

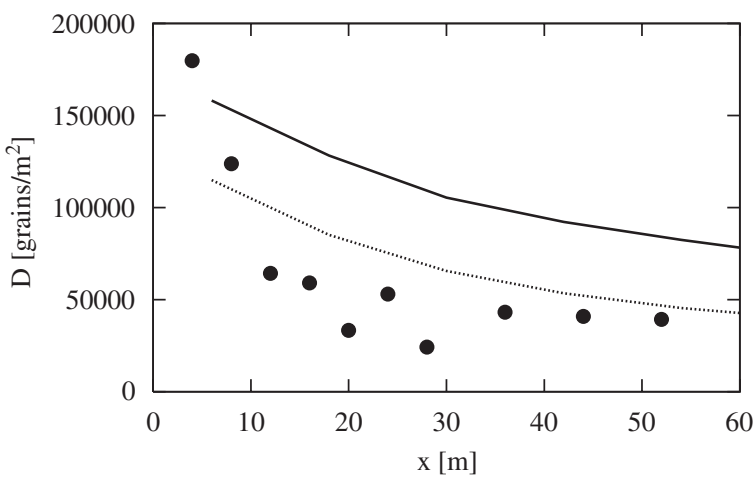

Fig. 12. Ground deposition downwind of the field along (a) north line and (b) south line. Experimental data (filled circles), LES simulation (solid line) and LES simulation corrected for smooth deposition (dotted line).

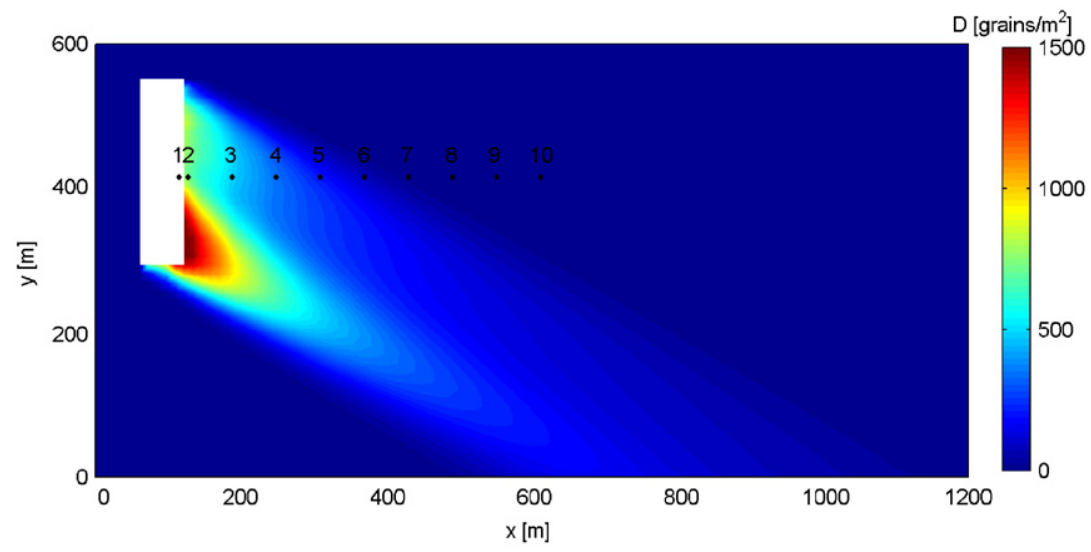

Fig. 13. Ground pollen deposition at the end of the simulation period.

In the framework proposed above, both quantities can be directly estimated from the simulation. In the numerical model used here, deposition on the grass field downwind is obtained by integrating in time the net pollen flux (there is no source so the net flux is the same as the deposition flux). Above the ragweed field, the net flux modeled by Eq. (6) is the difference between the emission and the deposition fluxes and its components can be obtained using Eqs. (9) and (10).

To illustrate the decomposition of the net deposition in its emission and total deposition parts as described above, the time integrated net flux is shown along the centerline of the ragweed field for the first $500 \mathrm{~m}$ of the domain in Fig. 14(b). The net flux is broken in its two components revealing a very large deposition within the field. The magnitude of deposition within the ragweed patch and downwind can be compared in the logarithmic scale plot presented in the insert in Fig. 14(b). Three different regions can be identified: a large deposition region corresponding to the ragweed patch, a slow decaying deposition region in the downwind field and a faster decaying deposition region which is caused by the effect of the finite size patch (in $y$ ). The sharp decrease in deposition on the edge of the field is surprisingly large. Deposition of particles to a vegetation canopy can be decomposed in settling, inertial impaction and Brownian diffusion (Raupach \& Lu, 2004) (the latter is negligible for pollen). The taller and denser canopy structure of the ragweed patch (compared to the mowed field) is responsible for the very large discrepancy between deposition inside and outside the patch. In the deposition model used here (Eq. (10)), the structure of the canopy is represented by the parameters $z_{0}$ and $d_{0}$. The large values of displacement height and roughness within the patch increase the deposition. Moreover, the increase in the friction velocity caused by the increased roughness, shown in Fig. 14(a), accentuates the difference even further (this is a secondary effect).

From the total emission fluxes it is possible to estimate the total number of pollen grains emitted from the field during the entire day. The result obtained is 62.61 billion grains, which gives an average emission of approximately 60,000 grains per plant. However, 37.79 billion grains (about 60\%) are deposited within the ragweed patch and the remaining 24.82 billion grains are available for cross-pollination. 
a

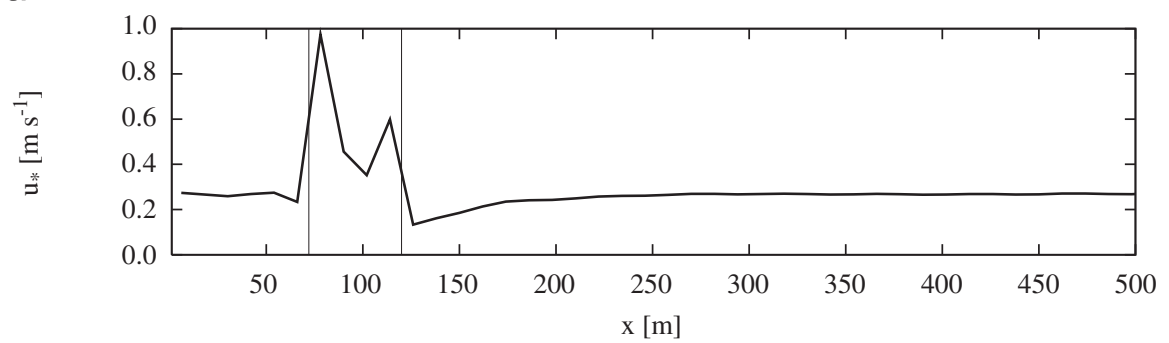

b

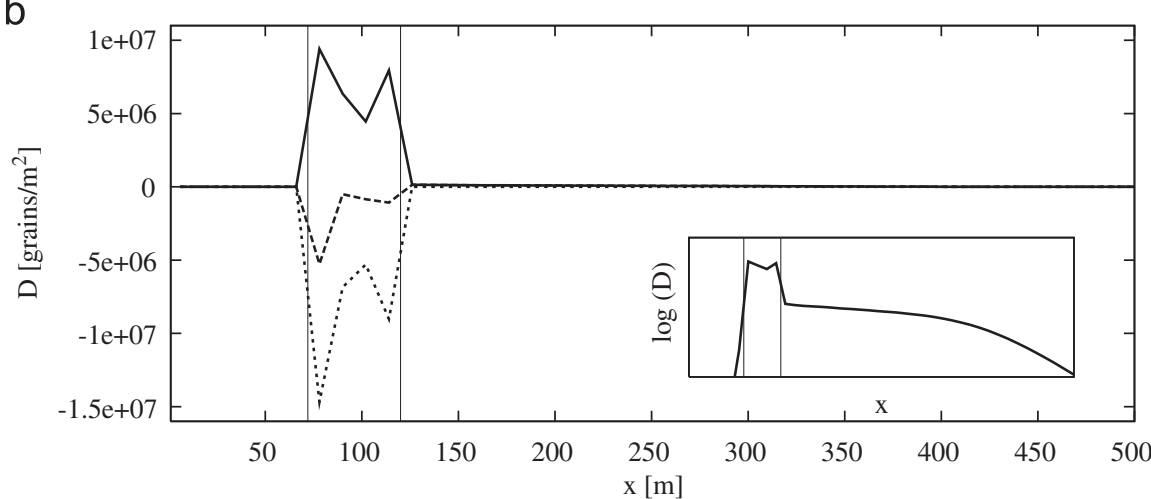

Fig. 14. (a) Time-averaged friction velocity along ragweed patch centerline, (b) time integrated net flux (dashed line), emission flux (dotted line) and deposition flux (solid line). Total deposition is shown in logarithmic scale in the insert. Vertical lines represent the edges of the ragweed patch.

Table 3

Kurtosis of instantaneous deposition flux at selected points indicated in Fig. 13

\begin{tabular}{|c|c|c|c|c|c|c|c|c|c|c|}
\hline Point & 1 & 2 & 3 & 4 & 5 & 6 & 7 & 8 & 9 & 10 \\
\hline Distance (m) & -6.00 & 6.00 & 66.00 & 126.00 & 186.00 & 246.00 & 306.00 & 366.00 & 426.00 & 486.00 \\
\hline Kurtosis & 3.44 & 3.28 & 3.22 & 2.95 & 2.92 & 3.06 & 10.18 & 40.82 & 120.35 & 217.19 \\
\hline
\end{tabular}

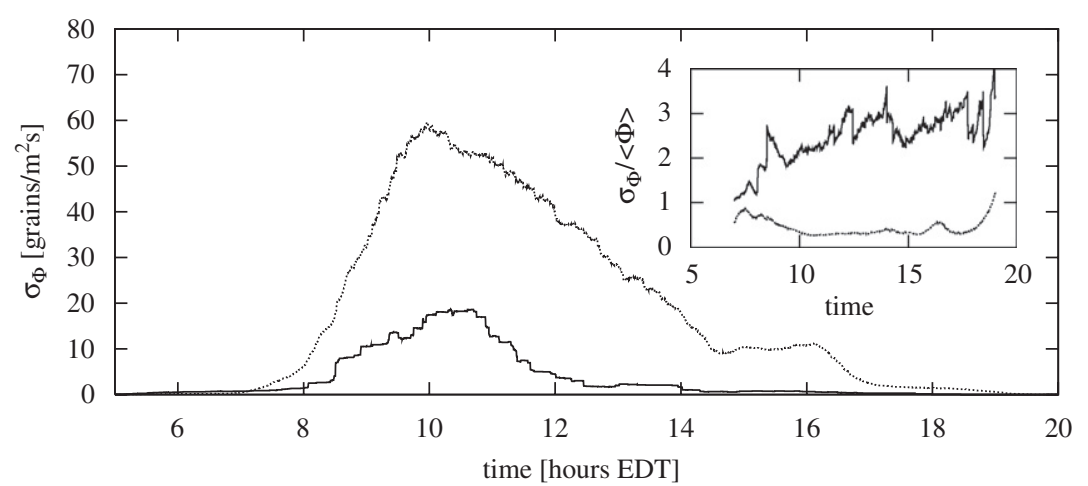

Fig. 15. Time evolution of RMS of instantaneous deposition $\left(\sigma_{\Phi}\right)$ at point 1 inside the ragweed patch (dotted line) and at point 8 outside (solid line) multiplied by 500 (see Fig. 13 for point locations). The fluctuation intensity is shown in the insert. In the figure $\Phi$ is used as short notation to $\Phi_{s g s}^{\text {dep }}$.

Another important issue which is often problematic to be studied experimentally is the occurrence of sporadic (but significant) deposition events far from the source field. These events are often responsible for significant gene flow even if the pollen fractions involved are fairly small (Ellstrand, 1992). In order to study these events, we look at the instantaneous deposition flux $\Phi_{\text {sgs }}^{\text {dep }}$ on 10 points indicated in Fig. 13 (the distance of the points to the trailing edge of the field is indicated in Table 3). Statistics were calculated using a running window of $1 \mathrm{~h}$. The time evolution of the root mean square (rms) of the flux fluctuations is shown for the points 1 and 8 in Fig. 15. The fluctuations are much larger for the point inside the ragweed field. This is consistent with the 
larger average deposition in the same region. However, the fluctuation intensity (i.e. the rms normalized by the mean deposition flux) is about one order of magnitude larger at point 8 (see the insert in Fig. 15). Fluctuation intensity grows monotonically as the distance form the edge of the field increases. The intermittency in the fluctuation time series (not shown) also increases with distance. This is clearly illustrated by the growth in the values of the kurtosis presented in Table 3, indicating that infrequent high peaks in deposition become more relevant.

\section{Discussion}

A framework to simulate pollen dispersal using LES has been developed, validated, and applied to better understand pollen dispersal in a recent field experiment. The pollen particles are modeled by a continuous Eulerian concentration field. A constant settling velocity is used to represent the gravitational effect. The effect of the canopy on the flow field is parameterized by two length scales (surface roughness and displacement height) incorporated into the lower boundary condition, eliminating the necessity of resolving the vertical height of the canopy, which would require a much finer grid.

The measurement of pollen concentration above the canopy, together with the use of the theoretical equilibrium profile derived by Chamecki et al. (2007), allows a simple and consistent approach to specify the pollen sources and sinks using the lower boundary condition. The same expression is used to derive boundary conditions over the grass field (sink) and over the emitting field (source and sink). A simple manipulation of the expression makes it possible to split the net flux above the canopy into its source and sink components. Finally, the material in the Appendix illustrates how the same theoretical profile can be used to deduce from the simulation what would be the deposition measured by smooth slides, providing a valuable tool for comparing experimental data to simulation results.

The main parameter governing the shape of the solution is the ratio between settling and turbulent transport $\alpha=S c_{T} w_{S} / \kappa u_{*}$. When $\alpha \gg 1$, the particles are so heavy that they follow a deterministic ballistic trajectory and are not affected by the turbulence. In the other extreme, when $\alpha \ll 1$, the settling effect is so small that the particles follow the fluid trajectories and the concentration field behaves as a passive scalar. Validation of the implementation against point source release experiments shows that the model is capable of reproducing the results with good accuracy over a wide range of values of $\alpha(\alpha=3.13$ for the Suffield case and $\alpha=0.11$ for the Brookhaven case). There are some open issues associated with predicting the horizontal spread of the pollen plume and whether this issue is related to mesoscale effects (meandering winds).

The application of the model to a natural field further validates the applicability of the approach to more complex problems and provides new insights in the interpretation of experimental data. In particular, the model provides an estimate of the total pollen emission and its deposition partition inside the field and downwind. It seems that the large amount of pollen deposited inside the ragweed field (about 60\%) is a good strategy to ensure successful pollination, since there is a large probability that pollen grains in this region will actually end up on female flowers. The pollen remaining airborne at the edge of the field (40\%) is critical to ensure cross-pollination and increases biodiversity. The low deposition rate on the grass demonstrates that some pollen will remain airborne at large distances from the field and probably be available for long-distance dispersal. Note that although $40 \%$ of the emitted pollen is airborne at the edge of the field, only $14 \%$ deposits inside the simulation domain (about $1000 \mathrm{~m}$ downwind) and 26\% remains airborne to be dispersed farther away. Further analysis of the deposition flux indicates the importance of sporadic intense events far from the source field.

Future directions should focus on the effects of atmospheric stratification on the dispersal distances and on controlled experiments to better understand the effects of field geometry and size on the partition between infield and cross-field deposition.

\section{Acknowledgments}

We acknowledge funding from the National Science Foundation biocomplexity Project BES-0119903 and EAR-0609690. We would also like to thank Mike Martin, Grace Brush, Chad Higgins, Mike Embry, and Stanley Featherstone for their collaboration in the field experiment.

\section{Appendix}

Deposition measurements obtained by placing greased microscope slides on the field are typically much smaller than the deposition on the field itself, mostly due to the smooth surface of the slide (e.g. Gregory, 1973; Raynor, Ogden et al., 1970). A simple relationship can be obtained between the simulated deposition on a rough surface and the deposition that would be measured by placing a smooth and small microscope slide on that surface. If the slide is very small compared to the relevant scale of the flow, one can safely assume that its presence does not affect the flow field (i.e. the mean velocity profile and the eddy diffusivity remain unchanged over the slide). The deposition can be modeled by Eq. (7), which can be written as

$$
\Phi_{s g s}^{d e p}(x, y)=-\beta w_{s} \widetilde{C}\left(x, y, z_{1}\right) \quad \beta=\frac{z_{1}^{\alpha}}{z_{1}^{\alpha}-z_{0, c}^{\alpha}} .
$$

The deposition on the rough surface is obtained by replacing $z_{0, c}$ by the momentum roughness $z_{0}$. However, for a smooth surface, $z_{0, c}$ should be replaced by the viscous length scale $v / u_{*}$, where $v$ is the kinematic viscosity of air. 
Under the stated assumptions, the concentration $\widetilde{C}\left(x, y, z_{1}\right)$ is not affected by the presence of the smooth slide and the ratio between the deposition on the real surface and on the smooth slide is given by

$$
\gamma=\frac{D_{\text {rough }}}{D_{\text {smooth }}}=\frac{\beta_{\text {rough }}}{\beta_{\text {smooth }}}=\frac{z_{1}^{\alpha}-\left(v / u_{*}\right)^{\alpha}}{z_{1}^{\alpha}-z_{0}^{\alpha}} .
$$

Note that this ratio is actually a function of $z_{0}, v, u_{*}, w_{s}$ and the height where the boundary condition is applied $z_{1}=d z / 2$. For the Suffield fine resolution simulation and the Brookhaven simulation the values are $\gamma=1$ and 3.10, respectively. Note that for the Suffield case there is no correction because the settling velocity is very high and this effect dominates the deposition. For the ragweed field simulation, the appropriate correction changes in time and space, since the friction velocity is not constant.

\section{References}

Albertson, J. D., \& Parlange, M. B. (1999). Surface length-scales and shear stress: Implications for land-atmosphere interaction over complex terrain. Water Resources Research, 35, 2121-2132.

Anfossi, D., Alessandrini, S., Castelli, S. T., Ferrero, E., Oettl, D., \& Degrazia, G. (2006). Tracer dispersion simulation in low wind speed conditions with a new 2D Langevin equation system. Atmospheric Environment, 40, 7234-7245.

Arritt, R. W., Clark, C. A., Goggi, A. S., Sanchez, H. L., Westgate, M. E., \& Riese, J. M. (2007). Lagrangian numerical simulations of canopy air flow effects on maize pollen dispersal. Field Crops Research, 102, 151-162.

Aylor, D. E. (2002). Settling speed of corn (Zea mays) pollen. Journal of Aerosol Science, 33, 1601-1607.

Aylor, D. E. (2005). Quantifying maize pollen movement in a maize canopy. Agricultural and Forest Meteorology, 131, $247-256$.

Aylor, D. E., \& Flesch, T. K. (2001). Estimating spore release rates using a Lagrangian stochastic simulation model. Journal of Applied Meteorology, 40, 1196-1208.

Aylor, D. E., Schultes, N. P., \& Shields, E. J. (2003). An aerobiological framework for assessing cross-pollination in maize. Agricultural and Forest Meteorology, 119, $111-129$.

Bouvet, T., \& Wilson, J. D. (2006). An approximate analytical solution for the deposition of heavy particles released from an elevated line source. Boundary-Layer Meteorology, 119, 1-18.

Bou-Zeid, E., Meneveau, C., \& Parlange, M. B. (2005). A scale-dependent Lagrangian dynamic model for large eddy simulation of complex turbulent flows. Physics of Fluids, 17, 025105

Chamberlain, A. C. (1967). Transport of Lycopodium spores and other small particles to rough surfaces. Proceedings of the Royal Society of London A, 296, 45-70.

Chamecki, M., Meneveau, C., \& Parlange, M. B. (2008). A hybrid spectral/finite-volume algorithm for large-eddy simulation of scalars in the atmospheric boundary layer. Boundary-Layer Meteorology, 128, 473-484.

Chamecki, M., van Hout, R., Meneveau, C., \& Parlange, M. B. (2007). Concentration profiles of particles settling in the neutral and stratified atmospheric boundary layer. Boundary-Layer Meteorology, 125, 25-38.

Dupont, S., Brunet, Y., \& Jarosz, N. (2006). Eulerian modelling of pollen dispersal over heterogeneous vegetation canopies. Agricultural and Forest Meteorology, 141, $82-104$.

Ellstrand, N. C. (1992). Gene flow by pollen: Implications for plant conservation genetics. Oikos, 63, 77-86.

Gaskell, P. H., \& Lau, A. K. C. (1988). Curvature-compensated convective transport: SMART, a new boundedness-preserving transport algorithm. International Journal for Numerical Methods in Fluids, 8, 617-641.

Germano, M., Piomelli, U., Moin, P., \& Cabot, W. H. (1991). A dynamic subgrid-scale eddy viscosity model. Physics of Fluids A, 3, $1760-1765$.

Gregory, P. H. (1973). Microbiology of the atmosphere. (2nd ed.), New York: Wiley, 377pp.

Hage, K. D. (1961). On the dispersion of large particles from a 15-m source in the atmosphere. Journal of Meteorology, 18, 534-539.

Helbig, N., Vogel, B., Vogel, H., \& Fiedler, F. (2004). Numerical modelling of pollen dispersion on the regional scale. Aerobiologia, 3, 3-19.

Högström, U. (1988). Non-dimensional wind and temperatures profiles in the atmospheric surface layer: a re-evaluation. Boundary-Layer Meteorology, 42, 55-78.

Honnay, O., Jacquemyn, H., Bossuyt, B., \& Hermy, M. (2005). Forest fragmentation effects on patch occupancy and population viability of herbaceous plant species. New Phytologist, 166, 723-736.

Jarosz, N., Loubet, B., Durand, B., Foueillassar, X., \& Huber, L. (2005). Variations in maize pollen emission and deposition in relation to microclimate. Environmental Science and Technology, 39, 4377-4384.

Jarosz, N., Loubet, B., Durand, B., McCartney, A., Foueillassar, X., \& Huber, L. (2003). Field measurements of airborne concentration and deposition rate of maize pollen. Agricultural and Forest Meteorology, 119, 37-51.

Jarosz, N., Loubet, B., \& Huber, L. (2004). Modelling airborne concentration and deposition rate of maize pollen. Atmospheric Environment, 38, 5555-5566.

Kaimal, J. C., \& Finnigan, J. J. (1994). Atmospheric boundary layer flows: Their structure and measurement. New York: Oxford University Press, 289pp.

Kind, R. J. (1992). One-dimensional aeolian suspension above beds of loose particles-a new concentration-profile equation. Atmospheric Environment A, 26, 927-931.

Kumar, V., Kleissl, J., Parlange, M. B., \& Meneveau, C. (2006). A large-eddy simulation of a diurnal cycle of the turbulent atmospheric boundary layer: Atmospheric stability and scaling issues. Water Resources Research, 42, W06D09.

Lehning, M., Löwe, H., Ryser, M., \& Raderschall, N. (2008). Inhomogeneous precipitation distribution and snow transport in steep terrain. Water Resources Research, 44, W07404.

Martin, M., Chamecki, M., Brush, G., Meneveau, C., \& Parlange, M. B. (2008). Pollen clumping and wind dispersal: Measurements, modeling and implications for genetic diversity. American Journal of Botany, to be submitted.

McCartney, H. A., \& Lacey, M. E. (1991). Wind dispersal of pollen from crops of oilseed rape (Brassica napus L.). Journal of Aerosol Science, 22, 467-477.

Moeng, C. H. (1984). A large-eddy-simulation model for the study of planetary boundary-layer turbulence. Journal of the Atmospheric Sciences, 41, $2052-2062$.

Piomelli, U., \& Balaras, E. (2002). Wall-layers models for large-eddy simulation. Annual Review of Fluid Mechanics, 34, 349-374.

Raupach, M. R., \& Lu, H. (2004). Representation of land-surface precesses in aeolian transport models. Environmental Modelling and Software, 19, 93-112.

Raynor, G. S., Hayes, J., \& Ogden, E. C. (1970). Experimental data on ragweed pollen dispersion and deposition from point and area sources. Technical Report 50224 (T-564), Brookhaven National Laboratory, 33pp.

Raynor, G. S., Hayes, J. V., \& Ogden, E. C. (1972). Dispersion and deposition of timothy pollen from experimental sources. Agricultural Meteorology, 9, 347-366.

Raynor, G. S., Ogden, E. C., \& Hayes, J. V. (1970). Dispersion and deposition of ragweed pollen from experimental sources. Journal of Applied Meteorology, 9, $885-895$.

Raynor, G. S., Ogden, E. C., \& Hayes, J. V. (1972). Dispersion and deposition of corn pollen from experimental sources. Agronomy Journal, $64,420-427$.

Shao, Y. (2001). Physics and modelling of wind erosion. (1st ed.), Berlin: Springer, 408pp.

Vickers, D., Mahrt, L., \& Belusic, D. (2007). Particle simulations of dispersion using observed meandering and turbulence. Acta Geophysica, 56, 234-256.

Walker, E. R. (1965). A particulate diffusion experiment. Journal of Applied Meteorology, 4, 614-621.

Wilson, J. D. (2000). Trajectory models for heavy particles in atmospheric turbulence: Comparison with observations. Journal of Applied Meteorology, 39, $1894-1912$.

Xie, Z., Hayden, P., Voke, P. R., \& Robins, A. G. (2004). Large-eddy simulation of dispersion: Comparison between elevated and ground-level sources. Journal of Turbulence, 5, 1-16. 